\title{
The ordering on permutations induced by continuous maps of the real line
}

\author{
CHRIS BERNHARDT \\ Department of Mathematics, Lafayette College, Easton, PA 18042, USA
}

(Received 12 May 1986 and revised 27 August 1986)

\begin{abstract}
Continuous maps from the real line to itself give, in a natural way, a partial ordering of permutations. This ordering restricted to cycles is studied.

Necessary and sufficient conditions are given for a cycle to have an immediate predecessor. When a cycle has an immediate predecessor it is unique; it is shown how to construct it. Every cycle has immediate successors; it is shown how to construct them.
\end{abstract}

\section{0 . Introduction}

Continuous maps from the real line to itself give, in a natural way, a partial ordering of permutations. The ordering, restricted to cycles, has been studied in [2] and [3].

In this paper attention is again restricted to cycles rather than to permutations in general; cycles correspond to periodic orbits. Necessary and sufficient conditions are given for a cycle to have an immediate predecessor. When a cycle has an immediate predecessor it is unique; the paper shows how to construct it. Every cycle has immediate successors; it is shown how to construct them.

Essentially, two cycles are next to one another in this ordering if and only if one can be obtained from the other by period doubling.

I would like to thank $Z$. Nitecki for some useful discussions during the formative stages of this paper. I would also like to thank the referee for helpful suggestions for the proof of Lemma 4.4 .

\section{Basics}

Throughout this paper $\left(S_{n},{ }^{\circ}\right)$ will denote the group of permutations on $n$ objects. $C_{n}$ denotes the subset of $S_{n}$ consisting of cycles. All functions will be assumed to be continuous maps from the real line to itself.

Many of the definitions used in this paper were first introduced in [3]. They are re-stated here for ease of exposition. A longer list of references for the ideas introduced here can be found in [3]. Much of the language used is algebraic; [1] may be helpful in giving a more geometric viewpoint.

Definition 1.1. Given a function, $f$, its set of permutations denoted Perm $(f)$ is defined by the following. A permutation, $\theta$, belongs to $\operatorname{Perm}(f)$ if there exist real $x_{1}<$ $x_{2}<\cdots<x_{n}$ such that $f\left(x_{i}\right)=x_{\theta(i)}$.

Definition 1.2. Let $\theta$ and $\eta$ be cycles. Say $\theta$ dominates $\eta$, denoted by $\eta \triangleright \theta$, if $\{f \mid \theta \in \operatorname{Perm}(f)\}$ is contained in $\{f \mid \eta \in \operatorname{Perm}(f)\}$. 
Definition 1.3. If $\theta$ is a cycle let $\operatorname{Dom}(\theta)$ denote the set of cycles dominated by $\theta$ i.e. $\operatorname{Dom}(\theta)=\{\eta \mid \eta \triangleright \theta\}$.

Definition 1.4. We will say $\theta$ is an immediate successor to $\eta$, or $\eta$ is an immediate predecessor to $\theta$, if $\theta \neq \eta$ and $\operatorname{Dom}(\theta)=\operatorname{Dom}(\eta) \cup\{\theta\}$.

Definition 1.5. Suppose that $\theta$ belongs to Perm $(f)$ and that $x_{1}, \ldots, x_{n}$ represents the reals such that $f\left(x_{i}\right)=x_{\theta(i)}$. Then a directed graph can be associated to $\theta$ and $f$ in the following way. The graph has $n-1$ vertices $J_{1}, \ldots, J_{n-1}$, and an arrow is drawn from $J_{k}$ to $J_{l}$ if and only if $f\left(\left[x_{k}, x_{k+1}\right]\right) \supseteq\left[x_{l}, x_{i+1}\right]$. This graph will be called the Markov graph associated to $f$ and $\theta$.

Definition 1.6. Given a permutation $\theta$ belonging to $S_{n}$ the primitive function $\bar{f}$ associated to $\theta$ is defined by the following:

(1) $\bar{f}(k)=\theta(k)$

(2) $\bar{f}(t k+(1-t)(k+1))=t \theta(k)+(1-t) \theta(k+1)$;

(3) $\bar{f}(x)=\theta(1)$ if $x<1$;

(4) $\bar{f}(x)=\theta(n)$ if $x>n$;

where $k=1, \ldots, n$ and $0 \leq t \leq 1$.

Definition 1.7. The Markov graph associated to $\theta$ and its primitive function will be called the Markov graph of $\theta$.

For proof of the following lemma see [3] where it is also called Lemma 1.8 .

LEMMA 1.8. Let $\theta$ belong to $C_{n}$ and $\eta$ to $C_{m}$ and $\theta \neq \eta$. Then $\theta$ dominates $\eta$ if and only if the Markov graph of $\theta$ has a non-repetitive loop of length $m$ corresponding to $\eta$.

Definition 1.9. Let $J_{i_{1}} J_{i_{2}} \cdots J_{i_{n}}$ denote a non-repetitive loop in the Markov graph of a permutation. This corresponds to a periodic point of period $n$. Let Cycle $\left(J_{i_{1}} J_{i_{2}} \cdots J_{i_{n}}\right)$ denote the element of $C_{n}$ that corresponds to this periodic point. From the fact that the primitive function is piecewise monotone it follows that Cycle $\left(J_{i_{1}} J_{i_{2}} \cdots J_{i_{n}}\right)$ is well-defined.

Example 1.10. Consider the cycle (123). It has Markov graph

$$
J_{1} \rightleftarrows \overrightarrow{J_{2}} \text {. }
$$

Figure 1 shows the graph of the primitive function associated to (123). This graph has $J_{1} J_{2} J_{2}$ as a loop of length three. Cycle $\left(J_{1} J_{2} J_{2}\right)$ is easily seen to be (123).

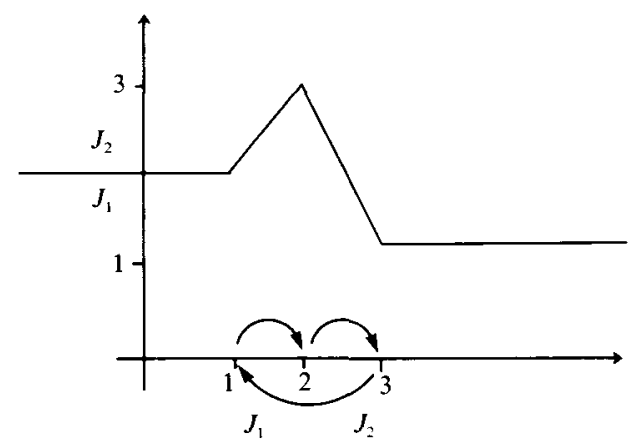

FIGURE 1 
Example 1.11. Consider the cycle ( $\left.\begin{array}{llll}1 & 3 & 2 & 4\end{array}\right)$. It has Markov graph

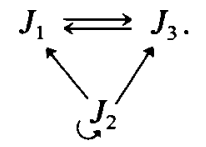

The only non-repetitive loops contained in this graph are $J_{1} J_{3}$ and $J_{2}$. Cycle $\left(J_{1} J_{3}\right)$ is (12) and Cycle $\left(J_{2}\right)$ is (1).

The aim of this paper is to prove the following theorem.

THEOREM 1.12. Let $\theta$ belong to $C_{n}$.

(1) The following statements are equivalent:

(a) $\theta$ has an immediate predecessor;

(b) $\theta$ is splittable;

(c) there is only one non-repetitive loop from $J_{1}$ to itself in the Markov graph of $\theta$;

(d) there does not exist a loop in the Markov graph of $\theta$ whose cycle is $\theta$.

(2) If $\theta$ has an immediate predecessor it is unique and is $\theta_{*}$.

(3) There are exactly $2^{n-1}$ immediate successors to $\theta$.

Remark 1.13. Some of the terms used in the above statement will be introduced later. However, it can be seen from statement 1 that the cycle in example 1.10 does not have an immediate predecessor and the cycle in example 1.11 does.

\section{Successors}

In this section it will be shown how to construct immediate successors to a cycle $\theta$. Later it will be shown that all successors can be constructed in this way.

Definition 2.1. Let $\theta \in C_{n}$, then $\theta^{*}$ is defined by $\theta^{*}(2 k)=2 \theta(k), \theta^{*}(2 k-1)=$ $2 \theta(k)-1$.

Definition 2.2. Let $\rho_{s}$ denote the transposition $(2 s-12 s)$.

Remark 2.3. If $\theta \in C_{n}$ then $\theta^{*} \circ \rho_{i_{1}} \circ \rho_{i_{2}} \circ \cdots \circ \rho_{i_{2 m-1}}$ belongs to $C_{2 n}$, where $1 \leq i_{j} \leq n$ for $1 \leq j \leq 2 m-1$. This is straightforward to check; or see [3].

LEMMA 2.4. If $\theta$ belongs to $C_{n}$ then $\theta^{*} \circ \rho_{i_{1}} \circ \rho_{i_{2}} \circ \cdots \circ \rho_{i_{2 m-1}}$ is an immediate successor to $\theta$, where $1 \leq i_{j} \leq n$ for $1 \leq j \leq 2 m-1$. The Markov graph of $\theta^{*} \circ \rho_{i_{1}} \circ \rho_{i_{2}} \circ \cdots \circ \rho_{i_{2 m-1}}$ contains only one non-repetitive loop from $J_{1}$ to itself.

Proof. Denote $\theta^{*} \circ \rho_{i} \circ \rho_{i_{2}} \circ \cdots \circ \rho_{i_{2 m-1}}$ by $\eta$. Consider the Markov graph of $\eta$. From the definition it is easily checked that there is exactly one arrow leaving $J_{2 \theta^{k}(1)-1}$ and it goes to $J_{2 \theta^{k+1}(1)-1}$. Thus there is a loop

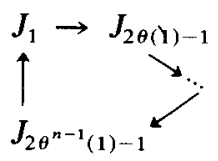

This is an attractive cycle in the sense that there may be arrows coming into this loop but there are no arrows leaving it. The subscripts in this loop contain all the odd numbers from 1 to $2 n-1$. Clearly, the cycle of this loop is $\theta$. 
Now consider the rest of the Markov graph of $\eta$. The only other loops must consist of $J$ 's with even subscripts. By definition there is an arrow from $J_{2 k}$ to $J_{2 m}$ iff one of the following holds: either

(1) $\eta(2 k) \leq 2 m$ and $\eta(2 k+1) \geq 2 m+1$; or

(2) $\eta(2 k+1) \leq 2 m$ and $\eta(2 k) \geq 2 m+1$.

Since $\eta(2 k)$ is either $2 \theta(k)$ or $2 \theta(k)-1$ and $\eta(2 k+1)$ is $2 \theta(k+1)-1$ or $2 \theta(k+1)$ there is an arrow from $J_{2 k}$ to $J_{2 m}$ iff one of the following holds: either

(1) $\theta(k) \leq m$ and $\theta(k+1) \geq m+1$; or

(2) $\theta(k+1) \leq m$ and $\theta(k) \geq m+1$,

but this is just the condition for there to be an arrow from $J_{k}$ to $J_{m}$ in the Markov graph of $\theta$. Thus the Markov graph of $\eta$ restricted to the $J$ 's with even subscripts is equivalent to the Markov graph of $\theta$. So $\operatorname{Dom}(\eta)=\operatorname{Dom}(\theta) \cup\{\eta\}$.

\section{The fundamental loop}

This section shows how to pick out a particular loop in the Markov graph of $\theta$. It is shown that the cycle of this loop is either $\theta$ or $\theta_{*}$. Later it will be shown that $\theta$ has immediate predecessors if and only if the cycle of the fundamental loop is $\theta_{*}$.

Definition 3.1. Let $\theta$ be a cycle of length $n$. Then the fundamental loop associated to $\theta$, denoted Loop $(\theta)$, is a path in the Markov graph of $\theta$ defined in the following way: Choose $\varepsilon$ small enough so that the length of the interval $\bar{f}^{i}([1,1+\varepsilon])$ is less than 1 for $0 \leq i \leq n$. Then for each $i$ there exists a unique integer $k(i)$ such that

$$
\bar{f}^{i}([1,1+\varepsilon]) \subseteq[k(i), k(i)+1] \text {. }
$$

Then Loop $(\theta)$ is the path defined by $J_{k(i)} \rightarrow J_{k(i+1)}$ for $0 \leq i \leq n+1$.

Note 3.2. Since $k(0)=1$ and $k(n)=1$ this path is actually a loop.

Definition 3.3. A cycle $\theta \in C_{2 n}$ will be called splittable if for each $k, 1 \leq k \leq n$, there exists a $j$ such that $f(\{2 k-1,2 k\})=\{2 j-1,2 j\}$.

Definition 3.4. If $\theta \in C_{2 n}$ is splittable then $\theta_{*}$ is defined by $\theta_{*}(k)=\operatorname{Int}\left[\frac{1}{2} \theta(2 k)\right]$, where Int means round up to the nearest integer.

Remark 3.5. In [3] $\theta_{*}$ was defined for simple permutations of power $2^{n}$.

Lemma 3.6. Let $\theta \in C_{n}$. Then Cycle $(\operatorname{Loop}(\theta))$ is either $\theta$ or $\theta_{*}$.

Proof. Loop $(\theta)$ is a loop of length $n$. This is either a non-repetitive loop or consists of repetitions of a shorter loop. These two cases will be considered separately.

If Loop $(\theta)$ consists of a non-repetitive loop then Cycle (Loop $(\theta)$ ) belongs to $C_{n}$. Since $\theta(i)$ is the unique integer in $\bar{f}^{i}([1,1+\varepsilon])$, where $[1,1+\varepsilon]$ is as in Definition 2.1 , it is clear that Cycle $(\operatorname{Loop}(\theta))$ is $\theta$.

Suppose Loop $(\theta)$ consists of repetitions of a shorter loop. As $\bar{f}^{i}([1,1+\varepsilon])$, $0 \leq i \leq n-1$, contains all the integers from 1 to $n$, Loop $(\theta)$ can repeat a vertex $J_{k}$ at most twice. Thus Cycle (Loop $(\theta)$ ) must be a cycle of length $n / 2$. The loop starts at $J_{1}$, this means $J_{1}$ is repeated twice and so $J_{2}$ cannot be repeated, thus $J_{2}$ is not in the path. Similarly, it can be shown that none of the even subscripted $J$ 's are in the loop and all of the odd subscripted $J$ 's are contained in the path. From this it follows easily that $\theta$ is splittable and Cycle $(\operatorname{Loop}(\theta))$ is $\theta_{*}$. 


\section{Predecessors}

This section shows when a cycle has an immediate predecessor. It also shows that if a cycle has an immediate predecessor then the predecessor is unique. A method of constructing it is also given.

LEMMA 4.1. If $\theta$ is splittable then $\theta_{*}$ is an immediate predecessor to $\theta$.

Proof. If $\theta$ is splittable then there exist transpositions $\rho_{i_{1}}, \ldots, \rho_{i_{2 m-1}}$ such that $\theta=\left(\theta_{*}\right)^{*} \circ \rho_{i_{1}} \circ \cdots \circ \rho_{i_{2 m-1}}$. Thus by Lemma $2.4 \theta_{*}$ is an immediate predecessor to $\theta$.

Remark 4.2. Note that if $\theta$ has an immediate predecessor then it is unique. This follows from the fact that $\triangleright$ is anti-symmetric (see [2] for proof of this).

LEMMA 4.3. Let $\theta \in C_{n}$. Let $J_{1} J_{2} \cdots J_{n}$ denote a loop in the Markov graph of $\theta$. If Cycle $\left(J_{1} J_{2} \cdots J_{n}\right)=\theta$ then Loop $(\theta)=J_{1} J_{2} \cdots J_{n}$.

Proof. Suppose that Cycle $\left(J_{1} J_{2} \cdots J_{n}\right)=\theta$ and that Loop $(\theta) \neq J_{1} J_{2} \cdots J_{n}$. This implies that there exists a periodic point $x$ with permutation type of $\theta$ with $\bar{f}^{i}(x) \in$ $(1, n)$ for any integer $i$. Thus whenever there is a periodic point with permutation type of $\theta$ there is another periodic point with permutation type of $\theta$ contained within it. This means there must be an infinite number of periodic points of period $n$.

Clearly one can choose a polynomial $P$ such that $P(i)=\theta(i)$ for $1 \leq i \leq n$. Since $P$ is a polynomial it only has a finite number of periodic points of period $n$. This gives a contradiction once it is seen that the Markov graph associated to $P$ contains in a natural way the Markov graph of $\theta$, (see [4]).

LEMMA 4.4. If Cycle (Loop $(\theta))=\theta$ then $\theta$ has no immediate predecessors and there is more than one non-repetitive loop from $J_{1}$ to itself in the Markov graph of $\theta$.

Proof. Loop $(\theta)$ gives a non-repetitive path from $J_{1}$, to itself of length $n$. However, since there is an integer $k, 1 \leq k \leq n-1$ such that $f^{k}(2)=1$ there is a path from $J_{1}$ to itself of length $k$, which has length strictly less than $n$. Thus the Markov graph of $\theta$ has more than one non-repetitive loop from $J_{1}$ to itself. For any positive integer $h$ let $\theta_{h n+k}$ be defined by taking the cycle of the loop that consists of going around Loop $(\theta) h$ times and then going around the shorter path once.

We make the following statements about $\theta_{h n+k}$.

(1) $\theta_{h n+k} \triangleright \theta$ for any $h$.

(2) $1<\theta_{h n+k}^{n}(1)<\theta_{h n+k}^{2 n}(1)<\cdots<\theta_{h n+k}^{h n}(1)$.

(3) $\theta_{h n+k}^{i n}(1)<\theta_{h n+k}^{j}$ (1) for all $i, j$ such that $0<j<h n, n+j, 0 \leq i \leq h-1$.

Proofs of the second two statements will be outlined. The first statement is obvious.

Let $\bar{f}$ denote the primitive function associated to $\theta$. Let $A$ denote the set of points in the interval $J_{1}$ whose trajectory of length $n$ follows Loop $(\theta)$. Then $A$ is an interval adjacent to 1 and is mapped by $\bar{f}^{n}$ linearly onto $J_{1}$. From this and the fact that $\bar{f}^{n}(1)=1$ statement 2 easily follows.

Let $x$ denote a point in $J_{1}$ whose orbit corresponds to $\theta_{h n+k}$. If $n \nmid j, 0<j<h n$ and $\bar{f}^{j}(x) \in J_{1}$ then $\bar{f}^{j}(x) \notin A$ (otherwise Loop $(\theta)$ would be repetitive). Therefore $\bar{f}^{j}(x)>\bar{f}^{i n}(x)$ for $i=0,1, \ldots, h-1$ and consequently the third statement is seen to be true. 
Suppose $\theta$ has an immediate predecessor, denote it $\eta$. Then $\theta_{h n+k} \triangleright \eta$ for any $h$. Suppose $\eta \in C_{m}$ and choose $h>m$. Now consider the loop of the Markov graph of $\eta$ that corresponds to $\theta_{h n+k}$. Denote it by $K_{i_{0}} K_{i_{1}} K_{i_{2}} \cdots$. Since $\theta_{h n+k}^{j n}(1)<\theta_{h n+k}^{(j+1) n}(1)$ one has $i_{j n} \leq i_{(j+1) n}$ for $j=0,1, \ldots, n-1$.

If $i_{j n}=i_{(j+1) n}$ for some value of $j$, then $K_{i_{j n}} K_{i_{j n+1}} \cdots K_{i_{(j+1) n}}$ is a loop. If this loop is non-repetitive then the cycle corresponding to this loop is an element of $C_{n}$ and it is easily seen (using an interval like $A$ in the argument above) that this cycle is $\theta$, but this gives a contradiction since it means $\eta$ dominates $\theta$.

So if $i_{j n}=i_{(j+1) n}$ then $K_{i_{j n}} K_{i_{j n+1}} \cdots K_{i_{j+1) n}}$ must consist of repetitions of a smaller loop. Denote the smaller loop by $K_{i_{j n}} K_{i_{j n+1}} \cdots K_{i_{j n+p}}$. Let $\bar{g}$ denote the primitive function for $\eta$ and let $B$ denote the interval constructed in the same way as $A$ above, but for this loop of length $p$. Then $\bar{g}^{p}$ maps $B$ linearly onto $J_{i_{j}}$. If $\left.\bar{g}^{p}\right|_{B}$ is order preserving then $\theta_{h n+k}^{j n+p}(1)<\theta_{h n+k}^{(j+1) n}(1)$, which contradicts statement 3 . If $\left.\bar{g}^{p}\right|_{B}$ is order reversing then since $\left.g^{P}\right|_{B}$ is expanding, $\theta_{h n+k}^{j n+2 p}(1)<\theta_{h n+k}^{j n}(1)$, which contradicts statement 2 if $n=2 p$, or statement 3 if $n>2 p$.

Thus $i_{j n} \neq i_{(j+1) n}$ for $j=0,1, \ldots, n-1$ and statement 2 gives $i_{0}<i_{n}<i_{2 n} \cdots<i_{h n}$ but this leads to a contradiction since-the Markov graph associated to $\eta$ has only $m-1$ vertices and by construction $m$ is less than $h$.

Thus $\eta$ cannot dominate $\theta_{h n+k}$ and so $\eta$ cannot be an immediate predecessor to $\theta$.

\section{Proof of Theorem 1.12}

Statement 1. Lemma 4.1 shows statement (b) implies (a). Lemmas 4.3, 4.4 show (a) implies (d). Lemma 3.6 shows (d) implies (b). Lemmas 4.3 and 4.4 show that (c) implies (d). Finally, Lemma 4.1 and Lemma 2.4 show that (b) implies (c).

Statement 2. This follows from remark 4.2, property (b) and Lemma 4.1.

Statement 3. Let $\theta$ belong to $C_{n}$. If $\eta$ is an immediate successor to $\theta$ then by statement $2, \theta=\eta_{*}$. Thus $\eta=\theta^{*} \circ \rho_{i_{1}} \circ \cdots \circ \rho_{i_{2 m-1}}$. Lemma 2.4 shows that any permutation formed by composing $\theta^{*}$ with an odd number of transpositions is an immediate successor to $\theta$. There are $2^{n-1}$ of these permutations.

\section{REFERENCES}

[1] L. Alseda, J. Llibre \& R. Serra. Minimal periodic orbits for continuous maps of the interval. Trans. Amer. Math. Soc. 286 (1984), 595-627.

[2] S. Baldwin. Generalizations of a theorem of Sarkovskii on orbits of continuous real-valued functions. Preprint.

[3] C. Bernhardt. Simple permutations with order a power of two. Ergod. Th. \& Dynam. Sys. (1984), 4, 179-186.

[4] C. W. Ho. On the structure of minimum orbits of periodic points for maps of the real line. Preprint. 